\title{
LARGE DEVIATIONS FOR RANDOM EVOLUTIONS WITH INDEPENDENT INCREMENTS IN THE SCHEME OF LÉVY APPROXIMATION
}

\author{
I.V. SAMOILENKO \\ Institute of Mathematics, \\ Ukrainian National Academy of Science, Kyiv, Ukraine, isamoil@imath.kiev.ua
}

November 17, 2018

Short title: LARGE DEVIATions FOR RANDOM EVOLUTiOnS

\begin{abstract}
In the work asymptotic analysis of the problem of large deviations for random evolutions with independent increments in the circuit of Lévy approximation is carried out. Large deviations for random evolutions in the circuit of Lévy approximation are determined by exponential generator for jumping process with independent increments.
\end{abstract}

KEY WoRDs: Lévy approximation, nonlinear exponential generator, Markov process, locally independent increments process, piecewise deterministic Markov process, singular perturbation.

Mathematics Subject Classification Primary: 60J55, 60B10, 60F17, 60K10; Secondary 60G46, 60G60.

\section{Introduction}

Asymptotic analysis of the problem of large deviations for random evolutions with independent increments in the circuit of Lévy approximation (see Koroliuk and Limnios, 2005, Ch. 9) is carried out in the paper.

Asymptotic analysis of random evolutions with independent increments in Lévy approximation scheme is conducted in the work of Koroliuk, Limnios and Samoilenko (2009).

In the monograph of Feng and Kurtz (2006) an effective method for studying the problem of large deviations for Markov processes is developed. It is based on the theory of convergence of exponential (nonlinear) operators. The exponential operator in the series scheme with a small series parameter $\varepsilon \rightarrow 0(\varepsilon>0)$ has the form (see, e.g. Koroliuk, 2011):

$$
\mathbb{H}^{\varepsilon} \varphi(x):=e^{-\varphi(x) / \varepsilon} \varepsilon \mathbb{L}^{\varepsilon} e^{\varphi(x) / \varepsilon},
$$

where the operators $\mathbb{L}^{\varepsilon}, \varepsilon>0$ define Markov processes $\zeta^{\varepsilon}(t), t \geq 0, \varepsilon>0$ in the series scheme on the standard phase-space $(G, \mathcal{G})$. Test-functions $\varphi(x) \in G$ are real-valued and finite. 
Random evolutions with independent increments (see Koroliuk and Limnios, 2005, Ch. 1) are given by:

$$
\xi(t)=\xi_{0}+\int_{0}^{t} \eta(d s ; x(s)), t \geq 0 .
$$

Markov processes with independent increments $\eta(t ; x), t \geq 0, x \in E$, are defined in $\mathbb{R}$ and given by the generators

$$
\Gamma(x) \varphi(u)=\int_{\mathbb{R}}[\varphi(u+v)-\varphi(u)] \Gamma(d v ; x), x \in E, \varphi(u) \in \mathcal{B}_{\mathbb{R}} .
$$

Remark 1. The process in $\mathbb{R}^{d}, d>1$ may also be studied. See Remark 7 for more details.

Markov switching process $x(t), t \geq 0$, on a standard phase space $(E, \mathcal{E})$ is defined by the generator

$$
Q \varphi(x)=q(x) \int_{\mathbb{E}}[\varphi(y)-\varphi(x)] P(x, d y), x \in E, \varphi(u) \in \mathcal{B}_{E} .
$$

Thus, random evolution (1) is characterized by the generator of two-component Markov process $\xi(t), x(t), t \geq 0$ (see Koroliuk and Limnios, 2005, Ch. 2)

$$
\mathbb{L} \varphi(u, x)=Q \varphi(\cdot, x)+\Gamma(x) \varphi(u, \cdot) .
$$

The basic assumption about the switching Markov process is the following condition

C1: Markov process $x(t), t \geq 0$, is uniformly ergodic with the stationary distribution $\pi(A), A \in$ $\mathcal{E}$.

Let $\Pi$ be a projector onto null-subspace of reducible-invertible operator $Q$, defined in (2):

$$
\Pi \varphi(x)=\int_{E} \pi(d x) \varphi(x) .
$$

The following correlation is true

$$
Q \Pi=\Pi Q=0
$$

Potential operator $R_{0}$ has the following property (Koroliuk and Limnios, 2005, Ch. 1):

$$
Q R_{0}=R_{0} Q=\Pi-I \text {. }
$$

Remark 2. It follows from the last correlation that under solvability condition

$$
\Pi \psi=0
$$

Poisson equation

$$
Q \varphi=\psi
$$

has the unique solution

$$
\psi=R_{0} \varphi
$$

when $\Pi \varphi=0$.

Remark 3. Studying of limit properties of Markov processes is based at the martingale characterization of such processes, namely we should regard

$$
\mu_{t}=\varphi(x(t))-\varphi(x(0))-\int_{0}^{t} \mathbb{L} \varphi(x(s)) d s,
$$


where $\mathbb{L}$ is the generator that defines Markov process $x(t), t \geq 0$, on the standard phase-space $(E, \mathcal{E})$. It has a dense domain $\mathcal{D}(\mathbb{L}) \subseteq \mathcal{B}_{E}$, that contains continuous functions with continuous derivatives. Here $\mathcal{B}_{E}$ - Banach space of real-valued finite test-functions $\varphi(x) \in E$, endowed by the norm: $\|\varphi\|:=\sup _{x \in E}|\varphi(x)|$.

Large deviation theory is based on the studying of exponential martingale characterization (see Feng and Kurtz, 2006, Ch.1):

$$
\widetilde{\mu}_{t}=\exp \left\{\varphi(x(t))-\varphi(x(0))-\int_{0}^{t} \mathbb{H} \varphi(x(s)) d s\right\}
$$

is the martingale.

Here exponential nonlinear operator

$$
\mathbb{H} \varphi(x):=e^{-\varphi(x)} \mathbb{L} e^{\varphi(x)}, \varphi(x) \in \mathcal{B}_{E} .
$$

Equivalence of (3) and (4) follows from the correlations:

Proposition (see Ethier and Kurtz, 1986, p.66)

$$
\mu(t)=x(t)-\int_{0}^{t} y(s) d s
$$

is the martingale if and only if

$$
\widetilde{\mu}(t)=x(t) \exp \left\{-\int_{0}^{t} \frac{y(s)}{x(s)} d s\right\} \text { is the martingale. }
$$

We may assume that the domain $\mathcal{D}(\mathbb{L})$ contains constants, and if $\varphi(x) \in \mathcal{D}(\mathbb{L})$, then there exists a constant $c$ such that $\varphi(x)+c \in \mathcal{D}(\mathbb{L})$ is positive.

Remark 4. The large deviation problem is realized in four stages (Feng and Kurtz, 2006, Ch.2):

1) Verify the convergence of the exponential (nonlinear) generator that defines large deviations;

2) Verify the exponential tightness of Markov processes;

3) Verify the comparison principle for the limit exponential generator;

4) Construct a variational representation for the limit exponential generator.

The stages 2)-4) for the exponential generator corresponding to the processes with independent increments are realized in Feng and Kurtz (2006). Some of the stages are also presented in the monograph of Freidlin and Wentzel (1998), where the large deviation problem is studied with the use of cumulant of the process with independent increments. Cumulant and exponential generator are obviously connected.

Really, generator of Markov process may be written in the form (see, e.g. Skorokhod, 1989)

$$
\mathbb{L} \varphi(x)=\int_{\mathbb{R}} e^{\lambda x} a(\lambda) \bar{\varphi}(\lambda) d \lambda,
$$

where $a(\lambda)$ - cumulant of the process, $\bar{\varphi}(\lambda)=\int_{\mathbb{R}} e^{-\lambda x} \varphi(x) d x$.

Inverse transformation gives

$$
\int_{\mathbb{R}} e^{-\lambda x} \mathbb{L} \varphi(x) d x=a(\lambda) \bar{\varphi}(\lambda) .
$$

Let's rewrite

$$
\int_{\mathbb{R}} e^{-\lambda x} \mathbb{L} \varphi(x) d x=\int_{\mathbb{R}} e^{-\lambda x} a(\lambda) \varphi(x) d x,
$$


and by taking

$$
e^{-\lambda x} \varphi(x)=: \widetilde{\varphi}(x)
$$

we obtain

$$
\int_{\mathbb{R}} e^{-\lambda x} \mathbb{L} e^{\lambda x} \widetilde{\varphi}(x) d x=\int_{\mathbb{R}} a(\lambda) \widetilde{\varphi}(x) d x
$$

Thus,

$$
e^{-\lambda x} \mathbb{L} e^{\lambda x}=a(\lambda)
$$

or, using the exponential generator:

$$
\mathbb{H} \varphi_{0}(x)=a(\lambda), \text { where } \varphi_{0}(x)=\lambda x .
$$

Our aim is to realize the stage 1) - to verify the convergence of the exponential (nonlinear) generator that defines large deviations $\mathbb{H}^{\varepsilon, \delta} \varphi_{\varepsilon}^{\delta}(u, x):=e^{-\varphi_{\varepsilon}^{\delta} / \varepsilon} \varepsilon \mathbb{L}_{\varepsilon}^{\delta} e^{\varphi_{\varepsilon}^{\delta} / \varepsilon}$ (see Theorem 1):

$$
\mathbb{H}^{\varepsilon, \delta} \varphi_{\varepsilon}^{\delta}(u, x) \rightarrow H^{0} \varphi(u), \varepsilon, \delta \rightarrow 0, \varepsilon^{-1} \delta \rightarrow 1 .
$$

To do this we use the method of solution of the problem of singular perturbation with two small series parameters.

Normalization of random evolution (1) by a small series parameters for solution of large deviation problem in Lévy approximation scheme is realized in a following way:

$$
\begin{gathered}
\xi_{\varepsilon}^{\delta}(t)=\xi_{\varepsilon}^{\delta}(0)+\int_{0}^{t} \eta_{\varepsilon}^{\delta}\left(d s ; x\left(s / \varepsilon^{3}\right)\right), t \geq 0, \\
\eta_{\varepsilon}^{\delta}(t)=\varepsilon \eta^{\delta}\left(t / \varepsilon^{3}\right), \\
\Gamma_{\varepsilon}^{\delta}(x) \varphi(u)=\varepsilon^{-3} \int_{\mathbb{R}}[\varphi(u+\varepsilon v)-\varphi(u)] \Gamma^{\delta}(d v ; x), x \in E,
\end{gathered}
$$

where $\varepsilon, \delta \rightarrow 0$ so that $\varepsilon^{-1} \delta \rightarrow 1$.

Remark 5. In the paper (Koroliuk, 2011) V.S.Koroliuk proposed to use the method of solution of the problem of singular perturbation for the studying of large deviations for random evolutions with independent increments in asymptotically small diffusion scheme.

In classical works asymptotical analysis of the problem of large deviations is made, as a rule, with the use of large series parameter $n \rightarrow \infty$, sometimes even some different parameters (see, e.g. Mogulskii, 1993).

The method, proposed in this work, with the use of two small parameters, was firstly realized in Samoilenko (2011) for the scheme of Poisson approximation.

\section{Lévy approximation conditions}

C2: Lévy approximation. The family of processes with independent increments $\eta^{\delta}(t ; x), x \in E$, $t \geq 0$ satisfies Lévy approxiation conditions:

LA1 Approximation of mean values:

$$
a_{\delta}(x)=\int_{\mathbb{R}} v \Gamma^{\delta}(d v ; x)=\delta a_{1}(x)+\delta^{2}\left[a(x)+\theta_{a}^{\delta}(x)\right],
$$

and

$$
c_{\delta}(x)=\int_{\mathbb{R}} v^{2} \Gamma^{\delta}(d v ; x)=\delta^{2}\left[c(x)+\theta_{c}^{\delta}(x)\right]
$$

where

$$
\sup _{x \in E}\left|a_{1}(x)\right| \leq a_{1}<+\infty, \sup _{x \in E}|a(x)| \leq a<+\infty, \sup _{x \in E}|c(x)| \leq c<+\infty .
$$


LA2 Asymptotic representation of intensity kernel

$$
\Gamma_{g}^{\delta}(x)=\int_{\mathbb{R}} g(v) \Gamma^{\delta}(d v ; x)=\delta^{2}\left[\Gamma_{g}(x)+\theta_{g}^{\delta}(x)\right]
$$

for all $g \in C_{3}(\mathbb{R})$ - measure-determining class of functions (see Jacod and Shiryaev, 1987, Ch. 7), $\Gamma_{g}(x)$ is a finite kernel

$$
\left|\Gamma_{g}(x)\right| \leq \Gamma_{g} \quad(\text { constant depending on } g) .
$$

Kernel $\Gamma^{0}(d v ; x)$ is defined on the measure-determining class of functions $C_{3}(\mathbb{R})$ by a relation

$$
\Gamma_{g}(x)=\int_{\mathbb{R}} g(v) \Gamma^{0}(d v ; x), \quad g \in C_{3}(\mathbb{R}) .
$$

Negligible terms $\theta_{a}^{\delta}, \theta_{c}^{\delta}, \theta_{g}^{\delta}$ satisfy the condition

$$
\sup _{x \in E}\left|\theta^{\delta}(x)\right| \rightarrow 0, \quad \delta \rightarrow 0 .
$$

LA3 Balance condition:

$$
\int_{E} \pi(d x) a_{1}(x)=0
$$

C3: Uniform square integrability:

$$
\lim _{c \rightarrow \infty} \sup _{x \in E} \int_{|v|>c} v^{2} \Gamma^{0}(d v ; x)=0 .
$$

C4: Exponential finiteness:

$$
\int_{\mathbb{R}} e^{p|v|} \Gamma^{\delta}(d v ; x)<\infty, \forall p \in \mathbb{R}
$$

\section{Main result}

Theorem 1. Solution of large deviation problem for random evolution

$$
\xi_{\varepsilon}^{\delta}(t)=\xi_{\varepsilon}^{\delta}(0)+\int_{0}^{t} \eta_{\varepsilon}^{\delta}\left(d s ; x\left(s / \varepsilon^{3}\right)\right), t \geq 0,
$$

defined by a generator of two-component Markov process $\xi(t), x(t), t \geq 0$

$$
\mathbb{L}_{\varepsilon}^{\delta} \varphi(u, x)=\varepsilon^{-3} Q \varphi(\cdot, x)+\Gamma_{\varepsilon}^{\delta}(x) \varphi(u, \cdot),
$$

where

$$
\Gamma_{\varepsilon}^{\delta}(x) \varphi(u)=\varepsilon^{-3} \int_{\mathbb{R}}[\varphi(u+\varepsilon v)-\varphi(u)] \Gamma^{\delta}(d v ; x), x \in E
$$

is realized by the exponential generator

$$
\begin{gathered}
H^{0} \varphi(u)=\left(\widetilde{a}-\widetilde{a}_{0}\right) \varphi^{\prime}(u)+\frac{1}{2} \sigma^{2}\left(\varphi^{\prime}(u)\right)^{2}+\int_{\mathbb{R}}\left[e^{v \varphi^{\prime}(u)}-1\right] \widetilde{\Gamma}^{0}(d v), \\
\widetilde{a}=\Pi a(x)=\int_{E} \pi(d x) a(x), \widetilde{a}_{0}=\Pi a_{0}(x)=\int_{E} \pi(d x) a_{0}(x), a_{0}(x)=\int_{\mathbb{R}} v \Gamma^{0}(d v ; x),
\end{gathered}
$$




$$
\begin{gathered}
\widetilde{c}=\Pi c(x)=\int_{E} \pi(d x) c(x), \widetilde{c}_{0}=\Pi c_{0}(x)=\int_{E} \pi(d x) c_{0}(x), c_{0}(x)=\int_{\mathbb{R}} v^{2} \Gamma^{0}(d v ; x), \\
\sigma^{2}=\left(\widetilde{c}-\widetilde{c}_{0}\right)+2 \int_{E} \pi(d x) a_{1}(x) R_{0} a_{1}(x), \widetilde{\Gamma}^{0}(v)=\Pi \Gamma^{0}(v ; x)=\int_{E} \pi(d x) \Gamma^{0}(v ; x) .
\end{gathered}
$$

Remark 6. Large deviations for random evolutions in Lévy approximation scheme are determined by exponential generator for jumping process with independent increments. Studying of large deviation problem for jumping process with independent increments is presented in monograph (Freidlin and Wentzel, 1998, Ch.3,4).

Remark 7. The limit exponential generator in the Euclidean space $\mathbb{R}^{d}, d>1$ is represented in the following view:

$H^{0} \varphi(u)=\sum_{k=1}^{d}\left(\widetilde{a}_{k}-\widetilde{a}_{k}^{0}\right) \varphi_{k}^{\prime}+\frac{1}{2} \sum_{k, r=1}^{d} \sigma_{k r} \varphi_{k}^{\prime} \varphi_{r}^{\prime}+\int_{\mathbb{R}^{d}}\left[e^{v \varphi^{\prime}(u)}-1\right] \widetilde{\Gamma}^{0}(d v), \varphi_{k}^{\prime}:=\partial \varphi(u) / \partial u_{k}, 1 \leq k \leq d$.

Here $\sigma^{2}=\left[\sigma_{k r} ; 1 \leq k, r \leq d\right]$ is the variance matrix.

In addition, the last exponential generator can be extended on the space of absolutely continuous functions (see Feng and Kurtz, 2006)

$$
C_{b}^{1}\left(R^{d}\right)=\left\{\varphi: \exists \lim _{|u| \rightarrow \infty} \varphi(u)=\varphi(\infty), \lim _{|u| \rightarrow \infty} \varphi^{\prime}(u)=0\right\} .
$$

Proof. Limit transition in the exponential nonlinear generator of random evolution is realized on the perturbed test-functions

$$
\varphi_{\varepsilon}^{\delta}(u, x)=\varphi(u)+\varepsilon \ln \left[1+\delta \varphi_{1}(u, x)+\delta^{2} \varphi_{2}(u, x)\right],
$$

where $\varphi(u) \in C^{3}(\mathbb{R})$ (the space of continuous bounded functions with continuous bounded derivatives up to third degree). Thus, we have from (5):

$$
\begin{gathered}
\mathbb{H}^{\varepsilon, \delta} \varphi_{\varepsilon}^{\delta}=e^{-\varphi_{\varepsilon}^{\delta} / \varepsilon} \varepsilon \mathbb{L}_{\varepsilon}^{\delta} e^{\varphi_{\varepsilon}^{\delta} / \varepsilon}=e^{-\varphi_{\varepsilon}^{\delta} / \varepsilon}\left[\varepsilon^{-2} Q+\varepsilon \Gamma_{\varepsilon}^{\delta}(x)\right] e^{\varphi_{\varepsilon}^{\delta} / \varepsilon}= \\
e^{-\varphi / \varepsilon}\left[1+\delta \varphi_{1}+\delta^{2} \varphi_{2}\right]^{-1}\left[\varepsilon^{-2} Q+\varepsilon \Gamma_{\varepsilon}^{\delta}(x)\right] e^{\varphi / \varepsilon}\left[1+\delta \varphi_{1}+\delta^{2} \varphi_{2}\right] .
\end{gathered}
$$

To obtain the asymptotic behavior of the last exponential generator we use the following results.

Lemma 1. Exponential generator

$$
H_{Q}^{\varepsilon} \varphi_{\varepsilon}^{\delta}(u, x)=e^{-\varphi_{\varepsilon}^{\delta} / \varepsilon} \varepsilon^{-2} Q e^{\varphi_{\varepsilon}^{\delta} / \varepsilon}
$$

has the following asymptotic representation

$$
H_{Q}^{\varepsilon} \varphi_{\varepsilon}^{\delta}=\varepsilon^{-1} Q \varphi_{1}+Q \varphi_{2}-\varphi_{1} Q \varphi_{1}+\theta_{Q}^{\varepsilon, \delta}(x)
$$

where $\sup _{x \in E}\left|\theta_{Q}^{\varepsilon, \delta}(x)\right| \rightarrow 0, \varepsilon, \delta \rightarrow 0$.

Proof. We have:

$$
\begin{gathered}
\mathbb{H}_{Q}^{\varepsilon} \varphi_{\varepsilon}^{\delta}=e^{-\varphi / \varepsilon}\left[1+\delta \varphi_{1}+\delta^{2} \varphi_{2}\right]^{-1} \varepsilon^{-2} Q e^{\varphi / \varepsilon}\left[1+\delta \varphi_{1}+\delta^{2} \varphi_{2}\right]= \\
{\left[1-\delta \varphi_{1}+\delta^{2} \frac{\varphi_{1}^{2}+\delta \varphi_{1} \varphi_{2}-\varphi_{2}}{1+\delta \varphi_{1}+\delta^{2} \varphi_{2}}\right]\left[\delta \varepsilon^{-2} Q \varphi_{1}+\delta^{2} \varepsilon^{-2} Q \varphi_{2}\right]=\delta \varepsilon^{-2} Q \varphi_{1}+\delta^{2} \varepsilon^{-2} Q \varphi_{2}-\delta^{2} \varepsilon^{-2} \varphi_{1} Q \varphi_{1}+\theta_{Q}^{\varepsilon, \delta}(x),}
\end{gathered}
$$

where

$$
\theta_{Q}^{\varepsilon, \delta}(x)=\delta^{3} \varepsilon^{-2} \frac{\varphi_{1}^{2}+\delta \varphi_{1} \varphi_{2}-\varphi_{2}}{1+\delta \varphi_{1}+\delta^{2} \varphi_{2}}\left[Q \varphi_{1}+\delta Q \varphi_{2}\right]-\delta^{3} \varepsilon^{-2} \varphi_{1} Q \varphi_{2}
$$


By the limit condition $\varepsilon^{-1} \delta \rightarrow 1, \varepsilon, \delta \rightarrow 0$, we finally have (9).

Lemma is proved.

Lemma 2. Exponential generator

$$
H_{\Gamma}^{\varepsilon, \delta}(x) \varphi_{\varepsilon}^{\delta}(u, x)=e^{-\varphi_{\varepsilon}^{\delta} / \varepsilon} \varepsilon \Gamma_{\varepsilon}^{\delta}(x) e^{\varphi_{\varepsilon}^{\delta} / \varepsilon}
$$

has the following asymptotic representation

$$
H_{\Gamma}^{\varepsilon, \delta}(x) \varphi_{\varepsilon}^{\delta}=H_{\Gamma}(x) \varphi(u)+\varepsilon^{-1} a_{1}(x) \varphi^{\prime}(u)+\theta_{\Gamma}^{\varepsilon, \delta}(x),
$$

where

$$
H_{\Gamma}(x) \varphi(u)=\left(a(x)-a_{0}(x)\right) \varphi^{\prime}(u)+\frac{1}{2}\left(c(x)-c_{0}(x)\right)\left(\varphi^{\prime}(u)\right)^{2}+\int_{\mathbb{R}}\left[e^{v \varphi^{\prime}(u)}-1\right] \Gamma^{0}(d v ; x),
$$

and $\sup _{x \in E}\left|\theta_{\Gamma}^{\varepsilon, \delta}(x)\right| \rightarrow 0, \varepsilon, \delta \rightarrow 0$.

Proof. We have:

$$
\begin{gathered}
\mathbb{H}_{\Gamma}^{\varepsilon, \delta}(x) \varphi_{\varepsilon}^{\delta}=e^{-\varphi / \varepsilon}\left[1+\delta \varphi_{1}+\delta^{2} \varphi_{2}\right]^{-1} \varepsilon \Gamma_{\varepsilon}^{\delta}(x) e^{\varphi / \varepsilon}\left[1+\delta \varphi_{1}+\delta^{2} \varphi_{2}\right]= \\
e^{-\varphi / \varepsilon}\left[1-\delta \varphi_{1}+\delta^{2} \frac{\varphi_{1}^{2}+\delta \varphi_{1} \varphi_{2}-\varphi_{2}}{1+\delta \varphi_{1}+\delta^{2} \varphi_{2}}\right]\left[\varepsilon \Gamma_{\varepsilon}^{\delta}(x) e^{\varphi / \varepsilon}+\varepsilon \delta \Gamma_{\varepsilon}^{\delta}(x) e^{\varphi / \varepsilon} \varphi_{1}+\varepsilon \delta^{2} \Gamma_{\varepsilon}^{\delta}(x) e^{\varphi / \varepsilon} \varphi_{2}\right]= \\
H_{\Gamma}^{\varepsilon, \delta}(x) \varphi(u)+e^{-\varphi / \varepsilon} \varepsilon \delta\left[\Gamma_{\varepsilon}^{\delta}(x) e^{\varphi / \varepsilon} \varphi_{1}-\varphi_{1} \Gamma_{\varepsilon}^{\delta}(x) e^{\varphi / \varepsilon}\right]+\widetilde{\theta}_{\Gamma}^{\varepsilon, \delta}(x),
\end{gathered}
$$

where

$$
\begin{gathered}
\widetilde{\theta}_{\Gamma}^{\varepsilon, \delta}(x)=\varepsilon \delta^{2}\left[e^{-\varphi / \varepsilon} \Gamma_{\varepsilon}^{\delta}(x) e^{\varphi / \varepsilon} \varphi_{2}-e^{-\varphi / \varepsilon} \varphi_{1} \Gamma_{\varepsilon}^{\delta}(x) e^{\varphi / \varepsilon} \varphi_{1}\right]+\varepsilon \delta^{2} \frac{\varphi_{1}^{2}+\delta \varphi_{1} \varphi_{2}-\varphi_{2}}{1+\delta \varphi_{1}+\delta^{2} \varphi_{2}}\left[e^{-\varphi / \varepsilon} \Gamma_{\varepsilon}^{\delta}(x) e^{\varphi / \varepsilon}+\right. \\
\left.e^{-\varphi / \varepsilon} \delta \Gamma_{\varepsilon}^{\delta}(x) e^{\varphi / \varepsilon} \varphi_{1}+e^{-\varphi / \varepsilon} \delta^{2} \Gamma_{\varepsilon}^{\delta}(x) e^{\varphi / \varepsilon} \varphi_{2}\right]-\varepsilon \delta^{3} e^{-\varphi / \varepsilon} \varphi_{1} \Gamma_{\varepsilon}^{\delta}(x) e^{\varphi / \varepsilon} \varphi_{2}
\end{gathered}
$$

We use the following results:

\section{Lemma 3.}

$$
\Gamma_{\varepsilon}^{\delta}(x) e^{\varphi(u) / \varepsilon} \varphi_{1}(u, x)=\varphi_{1}(u, x) \Gamma_{\varepsilon}^{\delta}(x) e^{\varphi(u) / \varepsilon}+(\varepsilon \delta)^{-1} \widehat{\theta}_{\Gamma}^{\varepsilon, \delta}(x),
$$

where for the negligible term $\sup _{x \in E}\left|\widehat{\theta}_{\Gamma}^{\varepsilon, \delta}(x)\right| \rightarrow 0, \varepsilon, \delta \rightarrow 0$.

Proof. Really, by (6) we have:

$$
\begin{gathered}
\Gamma_{\varepsilon}^{\delta}(x) e^{\varphi(u) / \varepsilon} \varphi_{1}(u, x)=\varepsilon^{-3} \int_{\mathbb{R}}\left[e^{\varphi(u+\varepsilon v) / \varepsilon} \varphi_{1}(u+\varepsilon v, x)-e^{\varphi(u) / \varepsilon} \varphi_{1}(u, x)\right] \Gamma^{\delta}(d v ; x)= \\
\varphi_{1}(u, x) \Gamma_{\varepsilon}^{\delta}(x) e^{\varphi(u) / \varepsilon}+(\varepsilon \delta)^{-1}\left[\varphi_{1}^{\prime}(u, x) \varepsilon^{-1} \delta \int_{\mathbb{R}} e^{\varphi(u+\varepsilon v) / \varepsilon} v \Gamma^{\delta}(d v ; x)\right] .
\end{gathered}
$$

Let's estimate the last integral. As soon as function $\varphi(u)$ is bounded, we have for fixed $\varepsilon$ :

$$
\int_{\mathbb{R}} e^{\varphi(u+\varepsilon v) / \varepsilon} v \Gamma^{\delta}(d v ; x)<e^{C} \int_{\mathbb{R}} v \Gamma^{\delta}(d v ; x)=\delta e^{C}\left[a_{1}(x)+\delta a(x)+\delta \theta_{a}^{\delta}(x)\right] .
$$

Thus, we see that the last term is negligible when $\varepsilon, \delta \rightarrow 0$.

Lemma is proved.

Lemma 4. Exponential generator

$$
H_{\Gamma}^{\varepsilon, \delta}(x) \varphi(u)=e^{-\varphi / \varepsilon} \varepsilon \Gamma_{\varepsilon}^{\delta}(x) e^{\varphi / \varepsilon}
$$


has the following asymptotic representation

$$
H_{\Gamma}^{\varepsilon, \delta}(x) \varphi_{\varepsilon}^{\delta}=H_{\Gamma}(x) \varphi(u)+\varepsilon^{-1} a_{1}(x) \varphi^{\prime}(u)+\theta^{\varepsilon, \delta}(x),
$$

where $\sup _{x \in E}\left|\theta^{\varepsilon, \delta}(x)\right| \rightarrow 0, \varepsilon, \delta \rightarrow 0$.

Proof. Let's rewrite (12), using the view of the generator (6). We have:

$$
H_{\Gamma}^{\varepsilon, \delta}(x) \varphi(u)=\varepsilon^{-2} \int_{\mathbb{R}}\left[e^{\Delta_{\varepsilon} \varphi(u)}-1\right] \Gamma^{\delta}(d v ; x),
$$

where

$$
\Delta_{\varepsilon} \varphi(u):=\varepsilon^{-1}[\varphi(u+\varepsilon v)-\varphi(u)] .
$$

We may rewrite it in a following way:

$$
\begin{aligned}
H_{\Gamma}^{\varepsilon, \delta}(x) \varphi(u)= & \varepsilon^{-2} \int_{\mathbb{R}}\left[e^{\Delta_{\varepsilon} \varphi(u)}-1-\Delta_{\varepsilon} \varphi(u)-\frac{1}{2}\left(\Delta_{\varepsilon} \varphi(u)\right)^{2}\right] \Gamma^{\delta}(d v ; x)+ \\
& \varepsilon^{-2} \int_{\mathbb{R}}\left[\Delta_{\varepsilon} \varphi(u)+\frac{1}{2}\left(\Delta_{\varepsilon} \varphi(u)\right)^{2}\right] \Gamma^{\delta}(d v ; x) .
\end{aligned}
$$

Easy to see that the function $\psi_{u}^{\varepsilon}(v)=e^{\Delta_{\varepsilon} \varphi(u)}-1-\Delta_{\varepsilon} \varphi(u)-\frac{1}{2}\left(\Delta_{\varepsilon} \varphi(u)\right)^{2}$ belongs to the class $C_{3}(\mathbb{R})$. Really,

$$
\psi_{u}^{\varepsilon}(v) / v^{2} \rightarrow 0, v \rightarrow 0
$$

Besides, this function is continuous and bounded for every $\varepsilon$ under the condition that $\varphi(u)$ is bounded. Moreover, the function $\psi_{u}^{\varepsilon}(v)$ is bounded uniformly by $u$ under the conditions C3, C4 and if $\varphi^{\prime}(u)$ is bounded.

Thus, we have:

$$
\begin{gathered}
H_{\Gamma}^{\varepsilon, \delta}(x) \varphi(u)=\varepsilon^{-2} \delta^{2} \int_{\mathbb{R}}\left[e^{\Delta_{\varepsilon} \varphi(u)}-1-\Delta_{\varepsilon} \varphi(u)-\frac{1}{2}\left(\Delta_{\varepsilon} \varphi(u)\right)^{2}\right] \Gamma^{0}(d v ; x)+ \\
\varepsilon^{-2} \int_{\mathbb{R}}\left[\Delta_{\varepsilon} \varphi(u)-v \varphi^{\prime}(u)-\varepsilon \frac{v^{2}}{2} \varphi^{\prime \prime}(u)\right] \Gamma^{\delta}(d v ; x)+\varepsilon^{-2} \delta a_{1}(x) \varphi^{\prime}(u)+\varepsilon^{-2} \delta^{2} a(x) \varphi^{\prime}(u)+ \\
\varepsilon^{-1} \delta^{2} c(x) \varphi^{\prime \prime}(u)+\varepsilon^{-2} \int_{\mathbb{R}}\left[\frac{1}{2}\left(\Delta_{\varepsilon} \varphi(u)\right)^{2}-\frac{v^{2}}{2}\left(\varphi^{\prime}(u)\right)^{2}\right] \Gamma^{\delta}(d v ; x)+\varepsilon^{-2} \delta^{2} \frac{1}{2} c(x)\left(\varphi^{\prime}(u)\right)^{2} .
\end{gathered}
$$

The functions in the second and third integrals are obviously belong to $C_{3}(\mathbb{R})$. Using Taylor's formula to the test-functions $\varphi(u) \in C^{3}(\mathbb{R})$, and condition PA2 we obtain:

$$
\begin{gathered}
H_{\Gamma}^{\varepsilon, \delta}(x) \varphi(u)=\varepsilon^{-2} \delta^{2} \int_{\mathbb{R}}\left[e^{v \varphi^{\prime}(u)}-1-v \varphi^{\prime}(u)-\frac{v^{2}}{2}\left(\varphi^{\prime}(u)\right)^{2}\right] \Gamma^{0}(d v ; x)+ \\
\varepsilon^{-2} \delta^{2} \int_{\mathbb{R}}\left(e^{v \varphi^{\prime}(u)} \varepsilon \frac{v^{2}}{2} \varphi^{\prime \prime}(\widetilde{u})-\varepsilon \frac{v^{2}}{2} \varphi^{\prime \prime}(\widetilde{u})-\varepsilon^{2} \frac{v^{4}}{8}\left(\varphi^{\prime \prime}(\widetilde{u})\right)^{2}\right) \Gamma^{0}(d v ; x)+ \\
\varepsilon^{-2} \delta^{2} \int_{\mathbb{R}} \varepsilon^{2} \frac{v^{3}}{3 !} \varphi^{\prime \prime \prime}(\widetilde{u}) \Gamma^{0}(d v ; x)+\varepsilon^{-2} \delta a_{1}(x) \varphi^{\prime}(u)+\varepsilon^{-2} \delta^{2} a(x) \varphi^{\prime}(u)+\varepsilon^{-1} \delta^{2} c(x) \varphi^{\prime \prime}(u)+ \\
\varepsilon^{-2} \delta^{2} \int_{\mathbb{R}} \varepsilon^{2} \frac{v^{4}}{4}\left(\varphi^{\prime \prime}(\widetilde{u})\right)^{2} \Gamma^{0}(d v ; x)+\varepsilon^{-2} \delta^{2} \frac{1}{2} c(x)\left(\varphi^{\prime}(u)\right)^{2} .
\end{gathered}
$$

By the limit condition $\varepsilon^{-1} \delta \rightarrow 1$, we finally have:

$$
H_{\Gamma}^{\varepsilon, \delta}(x) \varphi(u)=H_{\Gamma}(x) \varphi(u)+\varepsilon^{-1} a_{1}(x) \varphi^{\prime}(u)+\theta^{\varepsilon, \delta}(x),
$$


where $\sup _{x \in E}\left|\theta^{\varepsilon, \delta}(x)\right| \rightarrow 0, \varepsilon, \delta \rightarrow 0$.

Lemma is proved.

From Lemmas 3,4 we obtain

$$
H_{\Gamma}^{\varepsilon, \delta}(x) \varphi_{\varepsilon}^{\delta}=H_{\Gamma}(x) \varphi(u)+\varepsilon^{-1} a_{1}(x) \varphi^{\prime}(u)+\theta_{\Gamma}^{\varepsilon, \delta}(x),
$$

where $\sup _{x \in E}\left|\theta_{\Gamma}^{\varepsilon, \delta}(x)\right| \rightarrow 0, \varepsilon, \delta \rightarrow 0$.

Lemma is proved.

From (8) and (10) we see that

$$
\mathbb{H}^{\varepsilon, \delta} \varphi_{\varepsilon}^{\delta}=H_{Q}^{\varepsilon} \varphi_{\varepsilon}^{\delta}(u, x)+H_{\Gamma}^{\varepsilon, \delta}(x) \varphi_{\varepsilon}^{\delta}(u, x)
$$

Thus, using Lemmas 1,2, we obtain asymptotic representation

$$
\mathbb{H}^{\varepsilon, \delta} \varphi_{\varepsilon}^{\delta}=\varepsilon^{-1}\left[Q \varphi_{1}+a_{1}(x) \varphi^{\prime}(u)\right]+Q \varphi_{2}-\varphi_{1} Q \varphi_{1}+H_{\Gamma}(x) \varphi(u)+h^{\varepsilon, \delta}(x),
$$

where $h^{\varepsilon, \delta}(x)=\theta_{Q}^{\varepsilon, \delta}(x)+\theta_{\Gamma}^{\varepsilon, \delta}(x)$.

Now we may use the solution of singular perturbation problem for reducibly-invertible operator $Q$ (see Koroliuk and Limnios, 2005, Ch. 1).

$$
\begin{gathered}
Q \varphi_{1}+a_{1}(x) \varphi^{\prime}(u)=0 \\
Q \varphi_{2}-\varphi_{1} Q \varphi_{1}+H_{\Gamma}(x) \varphi(u)=H^{0} \varphi(u) .
\end{gathered}
$$

From the first equation we obtain

$$
\varphi_{1}(u, x)=R_{0} a_{1}(x) \varphi^{\prime}(u), \quad Q \varphi_{1}(u, x)=-a_{1}(x) \varphi^{\prime}(u) .
$$

After substitution to the second equation we have

$$
Q \varphi_{2}+a_{1}(x) R_{0} a_{1}(x)\left(\varphi^{\prime}(u)\right)^{2}+H_{\Gamma}(x) \varphi(u)=H^{0} \varphi(u)
$$

From the solvability condition:

$$
H^{0} \varphi(u)=\Pi H_{\Gamma}(x) \Pi \varphi(u)+\Pi a_{1}(x) R_{0} a_{1}(x) \mathbf{1}\left(\varphi^{\prime}(u)\right)^{2},
$$

where $\mathbf{1}$ is the unit vector.

Now, using (11) we finally obtain (7).

The negligible term $h^{\varepsilon, \delta}(x)$ may be found explicitly, using the solution of Poisson equation (see Remark 1, in details Koroliuk and Limnios, 2005)

$$
\varphi_{2}(u, x)=R_{0} \widetilde{H}(x) \varphi(u)-R_{0} a_{1}(x) R_{0} a_{1}(x) \mathbf{1}\left(\varphi^{\prime}(u)\right)^{2}, \quad \widetilde{H}(x):=H^{0}-H_{\Gamma}(x) .
$$

Theorem is proved.

\section{References}

[1] Ethier, S.N., Kurtz, T.G. (1986). Markov Processes: Characterization and convergence. New York: J. Wiley \& Sons.

[2] Feng, J., Kurtz, T.G. (2006). Large Deviation for Stochastic Processes. AMS, 131, RI.

[3] Freidlin, M.J., Wentzel, A.D. (1998). Random Perturbation of Dynamical Systems. Berlin: Springer-Verlag. 
[4] Jacod, J., Shiryaev, A.N. (1987). Limit Theorems for Stochastic Processes. Berlin: SpringerVerlang.

[5] Koroliuk, V.S., Limnios, N. (2005). Stochastic Systems in Merging Phase Space. WSP.

[6] Koroliuk, V.S., Limnios, N., Samoilenko, I.V. (2009). Lévy approximation of processes with locally independent increments with semi-Markov switching. Ukranian Math. Bull.6, No.3:343-356.

[7] Koroliuk, V.S. (2011). Large deviation problems for Markov random evolution with independent increments in the scheme of asymptotically small diffusion. Communications in Statistics - Theory and Methods 40(19-20):3385 - 3395.

[8] Mogulskii, A.A. (1993). Large deviation for processes with independent increments. Ann. Prob.21:202-215.

[9] Samoilenko, I.V. (2011). Large deviation for random evolutions with independent increments in the scheme of Poisson approximation (in Ukrainian). Prob. Theor. and Math. Stat.85 (to appear).

[10] Skorokhod, A.V. (1989). Asymptotic Methods in the Theory of Stochastic Differential Equations. Providence: AMS, v.78. 